
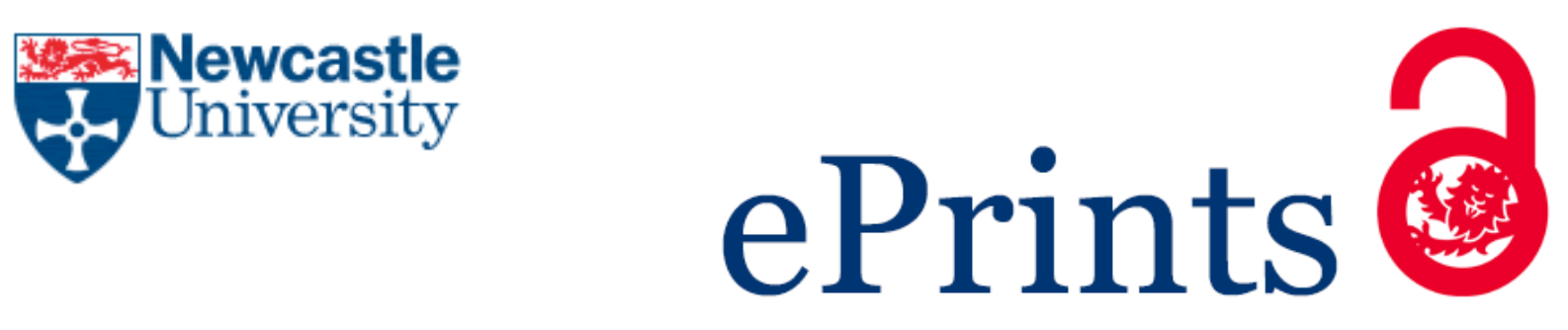

Parrish RR, Grady J, Codadu NK, Trevelyana AJ, Racca C.

Simultaneous profiling of activity patterns in multiple neuronal subclasses. Journal of Neuroscience Methods 2018

DOI: https://doi.org/10.1016/j.jneumeth.2018.03.012

\title{
Copyright:
}

(C) 2018. This manuscript version is made available under the CC-BY-NC-ND 4.0 license

DOI link to article:

https://doi.org/10.1016/j.jneumeth.2018.03.012

Date deposited:

$29 / 03 / 2018$

Embargo release date:

26 March 2019

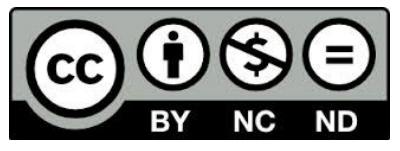

This work is licensed under a

Creative Commons Attribution-NonCommercial-NoDerivatives 4.0 International licence 


\section{Simultaneous profiling of activity patterns in multiple} 2 neuronal subclasses

3

4 R. Ryley Parrish ${ }^{\mathrm{a}}$, John Grady ${ }^{\mathrm{a}, \mathrm{b}}$, Neela K. Codadu ${ }^{\mathrm{a}}$, Andrew J. Trevelyan ${ }^{\mathrm{a} * *}$, 5 Claudia Racca*,

6 a Institute of Neuroscience, Medical School, Framlington Place, Newcastle upon Tyne, NE2 7 4HH, UK.

$8{ }^{\mathrm{b}}$ Kinghorn Centre for Clinical Genomics, Garvan Institute, 384 Victoria Street, Darlinghurst, 9 Sydney, NSW2010, Australia.

$10 *$ Corresponding authors (equal contribution)

Corresponding Authors:

13 C.R.: Institute of Neuroscience, Medical School, Framlington Place, Newcastle upon Tyne, NE2 4HH, UK; Tel. +44 (0)191 208 8716; claudia.racca@ncl.ac.uk

A.J.T.: Institute of Neuroscience, Medical School, Framlington Place, Newcastle upon Tyne, NE2 4HH, UK; Tel. +44 (0191) 208 5732; andrew.trevelyan@,ncl.ac.uk

\section{Highlights}

- We report a technique to profile activity patterns of multiple neuronal subclasses.

- $\mathrm{Ca}^{2+}$ imaging, patch-clamp, immunohistochemistry, and MATLAB profile cell activity.

- Using the technique, we follow a population of parvalbumin cells during a seizure. 
1 Abstract

2 Background: Neuronal networks typically comprise heterogeneous populations of

3 neurons. A core objective when seeking to understand such networks, therefore, is to 4 identify what roles these different neuronal classes play. Acquiring single cell 5 electrophysiology data for multiple cell classes can prove to be a large and daunting task. 6 Alternatively, $\mathrm{Ca}^{2+}$ network imaging provides activity profiles of large numbers of 7 neurons simultaneously, but without distinguishing between cell classes.

8 New Method: We therefore developed a strategy for combining cellular electrophysiology, $9 \mathbf{C a}^{2+}$ network imaging and immunohistochemistry to provide activity profiles for multiple 10 cell classes at once. This involves cross-referencing easily identifiable landmarks between 11 imaging of the live and fixed tissue, and then using custom MATLAB functions to realign 12 the two imaging data sets, to correct for distortions of the tissue introduced by the fixation 13 or immunohistochemical processing.

14 Results: We illustrate the methodology for analyses of activity profiles during 15 epileptiform events recorded in mouse brain slices. We further demonstrate the activity profile of a population of parvalbumin-positive interneurons prior, during, and following

17 a seizure-like event.

18 Comparison with Existing Methods: Current approaches to $\mathrm{Ca}^{2+}$ network imaging analyses are severely limited in their ability to subclassify neurons, and often rely on transgenic approaches to identify cell classes. In contrast, our methodology is a generic, affordable, and flexible technique to characterize neuronal behaviour with respect to classification based on morphological and neurochemical identity.

23 Conclusions: We present a new approach for analysing $\mathrm{Ca}^{2+}$ network imaging datasets, 24 and use this to explore the parvalbumin-positive interneuron activity during epileptiform events.

27 Keywords. Pyramidal neurons, GABAergic, Interneurons, Parvalbumin, Glia, Astrocytes, 28 Cortex, Seizure 


\section{1. Introduction}

A central aim of neuroscience is to understand how behaviour arises from the concerted contributions of individual neurons. This is a grand and non-trivial task, but an important first stage is documenting the firing and activity patterns of different classes of neurons and glia during different types of network events, in the various parts of the brain. $\mathrm{Ca}^{2+}$ network imaging is a technique that particular lends itself to this challenge, because it provides an opportunity to collect single-cell resolution data, recorded in real time, and often providing single-action potential temporal resolution (Cossart et al., 2003; MacLean et al., 2005; Smetters et al., 1999; Trevelyan et al., 2006; Yuste et al., 1992). Initially, this was achieved using the range of synthetic $\mathrm{Ca}^{2+}$ dyes developed by Roger Tsien and his colleagues (Grynkiewicz et al., 1985; Rodriguez et al., 2017) but latterly, there has been a switch to using genetically expressed $\mathrm{Ca}^{2+}$-indicators (GECIs) (Baird et al., 1999; Lin and Schnitzer, 2016; Miyawaki et al., 1997; Nakai et al., 2001).

Both synthetic dyes and GECIs have been used successfully to visualise network activity in many systems, to derive the activity profiles of up to several hundred neurons simultaneously, during particular network discharges (Lin and Schnitzer, 2016; Losi et al., 2017; Yang and Yuste, 2017). However, for both, there generally remains the task of subdividing these recordings to identify the activity profiles of specific subclasses of neurons. There are several ways in which one can try to do this. First, there are some model systems where cell-class specific conditional labelling is possible, the most obvious example being the use of Cre-recombinase expressed under specific promoters (Lewandoski, 2001; Orban et al., 1992; Sauer and Henderson, 1988). This is widely used for mouse models, but it must be appreciated how limited our options are, even for these model systems; only a tiny fraction of marker proteins can be utilised for conditional labelling, either through generating Cre $\mathrm{x}$ loxP mouse lines, or by injections of viral vectors for expression of promoter-driven labelling proteins, or Cre-driven, in mouse lines generated with cell class specific fluorescent reporters (Abe and Fujimori, 2013; Muzumdar et al., 2007; Powell et al., 2015). The technology is even more limited in other animal systems. The second option is to combine $\mathrm{Ca}^{2+}$ imaging with patch clamp recordings, as our lab and others have done (Losi et al., 2016; Trevelyan et al., 2007a; Trevelyan et al., 2006; Trevelyan et al., 2007b), but this immediately relinquishes the main benefit of network imaging, which is the instantaneous screening of large numbers of cells. 
To overcome these limitations, we developed a new strategy for matching landmarks

2 from the live and fixed imaging data sets, so that the activity profiles, derived from live imaging of hundreds of neurons as they participate in neuronal discharges, can then be matched to the post hoc immunohistochemical labelling. Since it is possible to raise antibodies against virtually any protein, and many antibodies are already commercially available, the potential for subclassifying $\mathrm{Ca}^{2+}$ network imaging data sets is almost unlimited. Furthermore, one can do this for multiple cell subclasses in parallel, in a single block of tissue. In this way, the firing patterns of any subclass of neuron, defined by a unique expression profile, can be derived, without the prior need for generating cell-specific conditional expression systems (mouse lines or viral vectors).

We present here details of our initial proof-of-principle demonstration using data collected from brain slices cross-matched to a permeabilisation technique of fixed tissue that allows immunohistochemical multiple labelling at least to the depth achievable in the live confocal imaging, thereby bypassing the need for reslicing the tissue. We discuss how this might be adapted to incorporate other new technical developments, including in vivo imaging and also tissue clearing. We also provide software for running a graphical-user interface (GUI) for matching the fixed and live imaging data sets, using the MATLAB platform.

\section{Material and methods}

2.1 Slice Preparation: Adult male C57BL/6 mice (ages 3 - 12 weeks) were used for these studies. All procedures performed on the mice were in accordance to the guidelines of the Home Office UK and Animals (Scientific Procedures) Act 1986. Mice were decapitated, with the brain being removed and stored in ice cold cutting solution containing (in $\mathrm{mM}$ ): $3 \mathrm{MgCl}_{2}$; $126 \mathrm{NaCl} ; 2.6 \mathrm{NaHCO}_{3} ; 3.5 \mathrm{KCl} ; 1.26 \mathrm{NaH}_{2} \mathrm{PO}_{4} ; 10$ glucose. $350 \mu \mathrm{m}$ coronal sections were made using a Leica VT1200 vibratome (Leica Microsystem, Germany) in the afore-mentioned cutting solution. Slices were then stored, at room temperature, in a submerged holding chamber for $1-4$ hours prior to experimentation. All the solutions were bubbled continuously to saturate with carboxygen $\left(95 \% \mathrm{O}_{2}\right.$ and $\left.5 \% \mathrm{CO}_{2}\right)$ in artificial cerebro-spinal fluid (aCSF) containing (in $\mathrm{mM}): 2 \mathrm{CaCl}_{2} ; 1 \mathrm{MgCl}_{2} ; 126 \mathrm{NaCl} ; 2.6 \mathrm{NaHCO}_{3} ; 3.5 \mathrm{KCl} ; 1.26 \mathrm{NaH}_{2} \mathrm{PO}_{4} ; 10$ glucose.

2.2 Dye loading: Oregon Green 488 BAPTA-1 (OGB1) - AM (50 $\mu \mathrm{g}$ vial; ThermoFisher) was mixed with $8 \mu \mathrm{l}$ of DMSO and $2 \mu \mathrm{l}$ of pluronic acid F-127 solution (10\% in DMSO; 
1 ThermoFisher). Meanwhile, the slices were preincubated at $37^{\circ} \mathrm{C}$ for $5 \mathrm{~min}$ in $3 \mathrm{ml}$ of aCSF with $8 \mu \mathrm{l}$ of Cremophor EL solution (0.5\% in DMSO; Sigma-Aldrich). The OGB1/pluronic acid/DMSO mixture was then added, and the slices were incubated for an additional $30-40$ min. The final concentrations were as follows: $12 \mu \mathrm{M}$ OGB1-AM, 0.6\% DMSO, 0.002\% Cremophor EL, and $0.01 \%$ pluronic F-127. The slices were then placed back in normal aCSF for at least $30 \mathrm{~min}$ before transferring to the recording chamber.

Astrocytic markers (e.g. sulphorhodamine 101, SR101) was introduced into the live perfusion solution, after completing the $\mathrm{Ca}^{2+}$ imaging data collection, to record astroctyte locations to enable matching of live and fixed imaging (Nimmerjahn et al., 2004). Briefly, SR101 (ThermoFisher) was dissolved in aCSF and washed over the slices in the recording chamber, at a concentration of $10 \mu \mathrm{M}$ for 1-5 minutes. The SR101 was then washed out of the slice for 10 minutes, prior to imaging the astrocytic staining.

2.3 Viral injections: C57BL/6 pups were injected with AAV5.Syn.GCaMP6f (cat. number AV-5-PV2822), purchased from the UPENN vector core. Injections were performed either on the day of birth (postnatal day 0, P0), or the following day (P1). Mice were set on a stereotaxic frame and anesthetized with isoflurane, following application of EMLA cream $(2.5 \%$ lidocaine and $2.5 \%$ prilocaine) to the left top of their head. A single injection site was made using a 10 $\mu 1$ Hamilton syringe, with a bevelled 36 gauge needle (World Precision Instruments). Injections were done at the following location: $\sim 1 \mathrm{~mm}$ anterior to lambda and $1 \mathrm{~mm}$ lateral to the midline into the left hemisphere, starting at $1.7 \mathrm{~mm}$ deep from the top of the dura mater, for a total of four separate $50 \mathrm{nl}$ injections, deepest first and coming up $0.3 \mathrm{~mm}$ for each subsequent injection. Approximately $200 \mathrm{nl}$ ( 1000 viral particles) were injected into the left hemisphere over a 10 min period. This resulted in widespread cortical expression of GCaMP6f into the neurons.

2.4 Electrophysiology: The electrophysiological data were collected using a Multiclamp 700B amplifier (Molecular Devices, USA) and pClamp software, digitized at 10kHz. The bath was mounted on a Scientifica movable top plate (Scientifica, UK) fitted with a heater plate (Warner Instruments, USA), and the incoming solution (perfusion at $1-3 \mathrm{ml} / \mathrm{min}$ ) was heated by a sleeve heater element (Warner Instruments). All imaging and electrophysiological recordings were done at $33-37^{\circ} \mathrm{C}$. Whole-cell patch-clamp recordings were made using 3-7 $\mathrm{M} \Omega$ pipettes (borosilicate glass; Harvard Apparatus, UK) controlled with Patchmaster micromanipulators (Scientifica). Pipettes were filled with a $\mathrm{KMeSO}_{4}$-based internal solution containing (in $\mathrm{mM}$ ): 
1314 (N-(2,6-dimethylphenylcarbamoylmethyl) triethylammoniumbromide), and $30 \mu \mathrm{m}$ Alexa

2 Fluor 568 or Alexa Fluor 594 (ThermoFisher). All experiments were run in aCSF without $\mathrm{Mg}^{2+}$

3 to induce epileptiform activity. The electrophysiological data were analysed off-line using

4 MATLAB (MathWorks. MA, USA).

$5 \quad 2.5$ Live Tissue Imaging: Live imaging was performed using two different spinning disk 6 confocal microscopes: (1) an Olympus DSU spinning disk BX/50WI upright microscope 7 (UMPlanFL N 20x, 0.50NA objective; Olympus, UK), illuminated using a Mercury arc lamp, 8 controlled with a fast Sutter shutter (Sutter Instrument, USA), using the standard FITC (for 9 OGB1 or GCaMP6f) and rhodamine (for Alexa Fluor 568 or 594) filter sets; (2) a spinning disc 10 confocal microscope (UMPlanFL N 20x, 0.50NA objective; VisiTech, UK). The tissue was 11 illuminated with a $491 \mathrm{~nm}$ laser (Cobolt Calypso 50; Cobolt, Sweden) for visualization of the 12 GCaMP6f, while the $561 \mathrm{~nm}$ laser (Cobolt Jive 50; Cobolt) was used to visualize the Alexa 13 Fluor 568, Alexa Fluor 594, and SR101 dyes. Both systems utilized Hamamatsu C9100 EM cameras (Hamamatsu Photonics, Japan) to collect images, run either by Simple PCI software (Digital Pixel, UK; microscope 1) or VoxCell software (Visitech; microscope 2), both installed on Dell Precision computers (Dell, UK). Further details of the sequence for collecting functional imaging data $\left(\mathrm{Ca}^{2+}\right.$ network imaging) and live imaging landmarks (dendritic structure and astrocyte distribution) are given in the results section. Off-line analysis of the images was performed using ImageJ (imagej.net, NIH) and in-house software implemented on MATLAB (MathWorks, USA).

21 2.6 Immunohistochemistry: The slices were fixed immediately after electrophysiological recording using 4\% paraformaldehyde (PFA) in phosphate buffered saline $(0.12 \mathrm{M}, \mathrm{pH} 7.4$; PBS). Slices were stored in fixative up to 7 days and then processed for fluorescence light microscopy and immunohistochemistry. Slices were then rinsed in PBS (2x10min each) and incubated in $50 \mathrm{mM} \mathrm{NH}_{4} \mathrm{Cl}$ for 20 mins in PBS to quench the free aldehyde groups. The slices were then rinsed in PBS (2x10min each) and blocked and permeabilised, in 1\% (v/v) Tween20 (P9416 Sigma, Merck) with 0.1\% (w/v) gelatine from bovine skin (G9382 Sigma) in PBS, for 3 hours, at room temperature. Slices were then incubated in combinations of primary antibodies (Table 1) in the same blocking/permeabilising solution, for 72 hours, at $4{ }^{\circ} \mathrm{C}$. Following this, sections were rinsed in PBS (3x10min each), then incubated in $0.1 \%$ Tween20 in PBS, for 3 hours at room temperature, with fluorescent streptavidin and secondary antibodies comprising combinations of the following list (depending on primary antibodies and 
1 avoidance of fluorescent spectrum interference): streptavidin Alexa Fluor 750 (1:1000); 2 donkey-anti-rabbit or guinea pig fluorescein isothiocyanate (FITC; 1:500) or Alexa Fluor 647 3 (1:1000) or 7-Amino-4-methylcoumarin-3-acetic acid-(AMCA, 1:100); goat-anti-rabbit or 4 guinea pig Alexa Fluor 647 (1:1000) or FITC (1:500); donkey anti-goat indodicarbocyanine 3 5 (Cy3, 1:1000); donkey anti-goat Cy5 (1:1000). All secondary antibodies were from Jackson 6 ImmunoResearch Labs (USA) but for the Alexa Fluor-conjugates and streptavidin 7 (ThermoFisher). At this time NeuroTrace 640/650 Deep-Red Fluorescent Nissl Stain (1: 0; 8 ThermoFisher) was also added to the incubation solution. Following this, sections were rinsed 9 with $0.1 \%$ Tween-20 in PBS ( $2 \times 10$ min each) and in PBS ( $2 \times 2$ hours each at room temperature).

10 The sections were then mounted on slides with ProLong Gold Antifade reagent (ThermoFisher). 11 Control experiments, in which the primary antibodies, NeuroTrace, and streptavidin were 12 omitted, gave no signal.

\begin{tabular}{|lllll|}
\hline Antibody & Concentration & Company & $\begin{array}{l}\text { Catalogue } \\
\text { Number }\end{array}$ & $\begin{array}{l}\text { NIF } \\
\text { Antibody ID }\end{array}$ \\
$\begin{array}{l}\text { Rabbit anti- } \\
\text { Parvalbumin }\end{array}$ & $1: 500$ & Swant & $\mathrm{PV}_{27}$ & AB 2631173 \\
\hline $\begin{array}{l}\text { Guinea Pig anti } \\
\text { Parvalbumin }\end{array}$ & $1: 500$ & Swant & $\mathrm{GP}_{72}$ & AB_2665495 \\
\hline $\begin{array}{l}\text { Goat anti- } \\
\text { Somatostatin }\end{array}$ & $1: 250$ & $\begin{array}{l}\text { Santa Cruz } \\
\text { Biotechnology/ }\end{array}$ & sc-7819 & AB_2302603 \\
\hline $\begin{array}{l}\text { Goat anti-OGB- } \\
\text { FITC/Alexa Fluor } \\
\text { 488 }\end{array}$ & $1: 100$ & ThermoFisher & A-11096 & AB_221558 \\
\hline Rabbit-anti-GFAP & $1: 250$ & & & \\
\hline
\end{tabular}

14 2.7 Fixed Imaging: Z stack, single or mosaic images were obtained by using a Zeiss ApotomeAxioImager 2 fluorescence microscope (Zeiss, UK) at 25x magnification. For analysis, optical 16 slices were reconstructed by using AxioVision (Zeiss) or ImageJ software. The original field 17 of view was located using the biocytin/Alexa Fluor filled cell, and a z-stack of images was 18 taken to ensure that the original plane of view and nearby surround planes of view were 19 recorded. The photomicrographs used in the figures are either single plane images from zstacks or were produced by first generating a maximum projection or extended depth of field 
1 of the z-stack by using ImageJ. The brightness and contrast of each image was then optimized, 2 and multi-panel figures were composed and labelled using Adobe Photoshop and Illustrator 3 (Adobe Systems).

\section{Results}

6

We set out to develop a methodology for deriving activity patterns simultaneously for multiple subclasses of neurons, using laboratory techniques that are easily implemented without the need for cell-specific conditional expression systems. The methodology is outlined schematically in Fig. 1. In short, the activity profiles were derived using $\mathrm{Ca}^{2+}$ network imaging, followed by imaging other fluorescently defined landmarks, which could then be matched up to the same landmarks identified after fixation and immunohistochemical processing. We describe each step in detail, below, exemplifying this using neocortical mouse brain slices bathed in epileptogenic aCSF.

\section{1 $\mathrm{Ca}^{2+}$ network imaging allows for the recording of hundreds of cells simultaneously}

Patch clamp recordings were made from neurons bathed in $\mathrm{Mg}^{2+}$-free aCSF (" $0 \mathrm{Mg}^{2+}$ model") (Gutierrez et al., 1999; Mody et al., 1987). Once the recording was established, we adjusted the field of view and plane of focus to optimise the number of cells labelled with the $\mathrm{Ca}^{2+}$ indicator (OGB1 or GCaMP6f), while retaining the recorded cell within that field of view. Neurons were recorded typically in voltage clamp mode, to identify bursts of synaptic bombardment indicative of pathophysiological network discharges, while simultaneously monitoring the wider network activity from the $\mathrm{Ca}^{2+}$ dye signal. This technique allows one to follow a single cell or group of cells over hours.

Previous work has shown that epileptiform activity evolves in a characteristic way after washing out $\mathrm{Mg}^{2+}$ ions, with several different discharge patterns identifiable from such patch clamp recordings (Trevelyan et al., 2006; Trevelyan et al., 2007b). We show an example, in Fig. 2 of the most extreme of these, a full ictal event, showing a typical tonic-clonic evolving structure, that propagated across the field of view.

In the example shown in Fig. $2 \mathrm{C}$, neurons were recruited to the ictal event over about a 10 s period (see progression from $t=15.8$ to $t=25.3 \mathrm{~s}$, Fig. $2 \mathrm{~B}$ ). Note however that in the period prior to this (the "pre-ictal period), the electrophysiological trace (Fig. 2A) showed a succession 
1 of large upward deflections (voltage clamp at $-30 \mathrm{mV}$, roughly half way between $\mathrm{E}_{\text {glut }}$ and $2 \mathrm{E}_{\mathrm{GABA}}$ ), when only a handful of cells were active (Figure $2 \mathrm{~B}, \mathrm{t}=0.63$ and $15.8 \mathrm{~s}$ ). Furthermore, 3 as the event continued into the clonic phase, one can identify cells that were recruited almost simultaneously, but then showed different kinetics of evolving $\mathrm{Ca}^{2+}$ signal.

While the $\mathrm{Ca}^{2+}$ imaging with single cell electrophysiology allows us to map specific cells during different network events, it does not allow us to determine the class or subclass of cells involved in the various events. Post hoc immunohistochemical labelling would enable this, if it were possible to match up the live imaging data set with views of the fixed immunohistochemical labelling. To achieve this, we needed a set of identifiable landmarks for matching the living and the fixed imaging data sets. The most useful of these are the unique branching pattern of the patch clamped cell, that was visualised by including both an Alexa dye (Fluor 568 or Fluor 594) for the live-imaging, and biocytin for visualising in the fixed tissue. When the recording electrode remained in situ, the Alexa dye within the electrode obscured the visualization of some of the important dendritic structures. Prior to imaging the dendritic structures, therefore, it is necessary to withdraw the electrode, taking care to achieve a good outside-out patch at the end (electrode withdrawn slowly, over $\sim 60$ s, while in voltage clamp mode, and continually monitoring the series resistance with a $10 \mathrm{mV}$ pulse), since this was presumed to equate to a similarly good resealing of the cell's membrane. If this was done, the cell remained visible, in a seemingly healthy state (absence of dendritic or axonal blebbing) long after the removal of the electrode, and allowed a set of matched z-stack images to be taken (Fig. 3A-B).

A second set of landmarks can be provided by visualising astrocytic processes using the specific live label, sulforhodamine (SR101). Since SR101 has similar excitation and emission spectra as for the Fluor dyes used for visualising the dendritic tree, we only introduced the SR101 after completing the z-stack views of the patch-clamp filled cell(s). There are, of course, other dyes (Alexa Fluor 350, cascade blue, for example) that could be used for visualising the dendritic tree, and which still retain spectral separation from the $\mathrm{Ca}^{2+}$ dyes, and we do not claim, in these proof-of-principle demonstrations, to have exhausted the methodological possibilities. Of note, SR101 also allows the identification of the active glia cells from the live imaging (see Fig. 4A). This will allow for post hoc identification of glia cells from neuronal cells. This should be done when using a calcium dye or a genetically encoded calcium sensor that is driven under a generic cell promoter (e.g. EF1alpha). 
Having taken 3 sets of z-stacks (1. $\mathrm{Ca}^{2+}$-dye labelling; 2. Dendritic structure of patched cell(s); 3. SR101-labelling of astrocytes), we took several low magnification images using a 2.5x magnification objective (Zeiss, Plan-NEOFLUAR; $0.075 \mathrm{NA}$ ). This provides an overview of the orientation of the slice within the live imaging set-up, as well as an approximate location of the imaged cell and field of view (Fig. 3C-D). We did this because we found that the process of matching the live and fixed tissue imaging data sets was made considerably easier if one started with an approximately co-aligned set of images.

\subsection{Post-fixation processing}

After completing the live imaging data collection (Fig. 4A), slices were fixed by immersing in 4\% PFA in PBS for immunohistochemistry. A total of 40 slices were fixed for immunohistochemistry, with 30 slices providing good immunohistochemical identification. Normally, when dealing with thick $(>300 \mu \mathrm{m})$ brain slices, these would be resectioned to achieve thin $(<50 \mu \mathrm{m})$ sections to allow good penetration of the antibodies. In this instance, we chose not to do this because otherwise, the matching of the live and fixed imaging data sets would need to be done separately for each resectioned slice, which would necessarily (by being themselves subsections of the original slice) have fewer matched landmarks than the intact slice. We therefore developed and optimised a strategy for permeabilising and blocking the thick tissue slices, as well as incubating the antibodies, which uses 1\% Tween-20 as detergent and $0.1 \%$ gelatine as blocking agent. Tween-20 was chosen over saponin and Triton-X100 because these were found to affect the tissue structure by being too mild and too harsh tissue permeabilisers, respectively, compared to Tween-20. Gelatine from bovine skin was chosen over bovine serum albumin, skimmed dried milk and serum as these were found to quench the fluorescence of the NeuroTrace. Slices were then labelled for pan-neuronal markers (e.g. NeuroTrace), glia markers (e.g. GFAP), biocytin for the patch-clamped cell (Streptavidin) and other cell classes (e.g. parvalbumin (PV), GAD67, or somatostatin (SOM)) as necessary for the experiment. This protocol provided immunohistochemical labelling at least as deep into the slice $(>100 \mu \mathrm{m})$ as the live confocal imaging.

Following immunohistochemistry labelling, slices were mounted on slides and the filled cell relocated. Typically, microscope stages dictate how the microscope slide is held, so it is helpful to bear this in mind when mounting the brain slice on to the microscope glass slide, in order to achieve an orientation close to that of the live imaging (see Fig. 3C-D). We next took z-stacks of the different fluorescent labelling, so as to incorporate the approximate field of view 
1 and plane of focus at which the live imaging of the activity patterns was performed for the $\mathrm{Ca}^{2+}$ network imaging. The live and fixed tissue imaging was then compared and matched. This required more than simply orienting the live and fixed imaging, because there were additional hurdles. First the tissue shrunk slightly (mean $11 \%$, range $5-30 \%, n=4$ ) during the fixation and staining process, and sometimes unevenly too. Further, because our live and fixed imaging systems had different mounted cameras, the resolution of the images was different. The final issue was to identify the correct plane of focus in the fixed tissue as for the time series data, which held the information about the cells' activity patterns.

To overcome these technical issues, we developed a custom-written, image-matching, graphical user interface (GUI; supplementary information, matchgui.m) implemented using MATLAB software. The first stage was to identify specific branch points of identified dendrites of the recorded neuron in both the live and fixed imaging data (Fig. 4). By working along a dendrite, one gradually built up a constellation of matching paired landmarks. These can also be supplemented by identifying matched glia if not enough other landmarks are present (Fig. 4Aii-4Bii). Ideally, one would like to have matching landmarks distributed throughout the entire live imaging field of view, because that represents the full spatial extent for which firing pattern data were collected. In reality, this often cannot be achieved. For example, in Fig. 4Aii the upper left quadrant of the live imaging was underrepresented with landmarks, and this led to reduced confidence in the matching algorithm in this segment. The glial landmarks have the potential to provide distributed landmarks, but as is apparent from Fig. 4Ai and Fig. $4 \mathrm{Bi}$, the discrete nature of the SR101 staining, which is mainly confined to the astrocyte cell bodies, is not always so easy to relate to the post-fixation GFAP labelling, which is found in both cell bodies and processes. The difficulty arises partly because the more complex SR101 and GFAP labellings provided fewer cues for ascribing a match, and secondly because the SR101 and the GFAP labellings on occasions appeared to show slightly different morphologies, even when we were quite confident about the spatial match (e.g. when in close proximity to a prominent dendritic landmark). Nevertheless, it was generally possible to achieve an extensive distribution of landmark pairs. Note, however, that it may still be possible to improve this further by patching multiple cells within the field of view. These cells need not necessarily be patched throughout the experiment, but rather, one could patch multiple cells sequentially, after the end of the $\mathrm{Ca}^{2+}$ network imaging data collection, simply for the purpose of enhancing the matching process using the more secure dendritic branching data. 
The designation of paired landmarks was done using custom software, implemented using MATLAB (matchgui.m code). This code provides a graphical user interface (GUI) for scrolling through the respective z-stacks (including sequential z-stacks taken using different filter sets), selecting confocal pairs of live and fixed images, and zooming in to examine particular features of the images together. Once all the paired landmark matches are entered, matchgui then creates a vector distortion, interpolated between the landmarks. This is implemented the same for all images from either the fixed or the live imaging data (note that only one set needs changing and the other remains the same). Since the intention was to then use these to interpret the $\mathrm{Ca}^{2+}$ network imaging data, we kept the living imaging data set constant, and distorted the fixed imaging data (Fig. 4Aiii,Biii,C).

We provide a full description of how to use the matchgui software in a parallel publication (Parrish et al., 2018), including more detail about finding the equivalent z-planes in the live and fixed images. The software provides three different transformation options (Figure 5): Linear Conformal (LC), Piecewise, and Linear Weighted Mean (LWM). The matches for all three are good in the central region of the image, where they are constrained by many landmark pairs, but towards the edge of the range of landmarks, they start to show errors, which are important to recognise. The Piecewise and LWM transformations both show distortions at the edge, and which, for the former, precludes any matching. In contrast, the LC transformation tends to maintain the local cellular positional relations, while increasingly being offset from the live imaging. The local constellations of cells, however, are still clearly identifiable, and can therefore still be used to ascribe cell class identities from the fixed data back onto the live imaging data set. Thus, these issues do not necessarily preclude the matching process, even towards the edge of the images, although they do preclude an easy quantification of the matching success.

The transformations produced extremely good correspondence between the living and fixed images over an extended field of view, and in the examples shown, allowed a mapping of more than 100 cells from the $\mathrm{Ca}^{2+}$ imaging to the fixed tissue (Figs. 4-6). This represents around $50-60 \%$ of the cells for which we had functional data, but note that the matching was close to $100 \%$ for the central region, where the matching process works best (Fig 6).

We repeated this process also for imaging using GECIs (Fig. 7). GCaMP6f labelling was achieved through injections of AAV viral vectors carrying the gene. Interestingly, in all our studies, tissue fixation did not fully quench the GCaMP6f signal (Fig. 7B). This allowed easier 
1 identification of multiple GCaMP6f labelled neurons between the live and fixed images (Fig. 2 7), but a downside was that this imaging bandwidth was unavailable for other 3 immunohistochemical analysis. Despite this limitation, we could still image across multiple 4 other wavelength bands, as illustrated in Figures 7 and 8, which shows staining for biocytin, 5 NeuroTrace, parvalbumin (PV, Fig 7), and somatostatin (SOM, Fig 8). The first two were 6 necessary for landmark matching, while the NeuroTrace also provides a pan-neuronal label, 7 and the PV-and SOM-immunohistochemistry labels an interesting subpopulation of cortical 8 interneurons. Figure 8 thus demonstrates how in principle the matching technique can be used 9 to obtain activity data from multiple neuronal classes.

Having identified specific subclasses of neurons using this methodology, we next applied this information to the $\mathrm{Ca}^{2+}$ network analyses (Fig. 9). The example shown included $7 \mathrm{PV}$ expressing interneurons present in both the live and fixed imaging (Fig. 7C and Fig. 9A), out of a total of 161 neurons which showed dynamically modulated $\mathrm{Ca}^{2+}$ signal (note that some very brightly fluorescent cells on occasions do not show modulation of fluorescence even during ictal activity, most probably because their high fluorescence was caused by pathological damage to the cells from the slice preparation). Our post hoc identification of this subpopulation of neurons could then be used to determine how they behaved during various electrographic events. In the example shown in Fig. 7 and Fig. 9, the PV cells were overrepresented in the initial discharges $(10.8 \%$ of early active neurons, as opposed to $4.3 \%$ of the total population). A second notable finding was that all the PV neurons showed firing throughout the ictal event, although some cells did not show increases in $\mathrm{Ca}^{2+}$ signal for every discharge. This may be because the calcium sensor was saturated already, or alternatively that the cell had gone into depolarising block. These results are consistent with the view that, whilst these interneurons may initially provide restraint of the ictal wavefront (Cammarota et al., 2013; Trevelyan and Schevon, 2013), this restraining action is short-lived, and that later, they may actually trigger the late discharges (Ellender et al., 2014; Fujiwara-Tsukamoto et al., 2003). 


\section{4. Discussion}

2 We present here new methodologies for enhancing the analysis of $\mathrm{Ca}^{2+}$ network imaging data.

3 The power of this technology lies in its facility for providing activity profiles of hundreds of neurons, with single cell resolution and video rate temporal resolution (Yuste et al., 1992). We can now sub-classify these hundreds of cells into their component neuronal classes. The cellspecificity is provided by post hoc immunohistochemical analyses, which can be utilized with synthetic live imaging dyes that have no particular cell-specificity (or viral vectors without specificity), and in animal lines without cell-specific molecular biological adaptations such as Cre-recombinase expression. Thus the methods can be applied to any animal system, as long as one can, first, introduce the dyes into the network, and second, raise, or have access to, specific antibodies to label up subpopulations of cells.

These experiments provide the initial proof-of-principle that it is possible to match the immunohistochemical data for large numbers of neurons onto the live imaging data, but there are several further ways in which this could yet be improved. For instance, one limitation with the data we show here is that we only had three fluorescent channels for labelling neurons, and one of these used a pan-neuronal stain, NeuroTrace. Another fluorescence channel was used to stain glial cells when using the $\mathrm{Ca}^{2+}$ dyes, which we used for matching purposes. However, we found that the glia did not provide as good a set of landmarks as the filled neurons, so an obvious improvement may be to do the landmark matching from multiple labelled neurons instead of just a single cell. This would free up a fluorescence channel for a different neuronal antibody. Another possibility would be to use tissue clearing techniques (e.g. CLARITY; iDISCO; for review see (Vigouroux et al., 2017)) with the live-fixed matching method, since these allow for immunohistochemistry and 3D imaging of tissue

Our analyses used two different $\mathrm{Ca}^{2+}$ indicators, OGB1 and GCaMP6f expressed under a general neuronal promoter. We imaged epileptiform discharges induced by removing $\mathrm{Mg}^{2+}$ ions. This preparation particularly lends itself to testing new $\mathrm{Ca}^{2+}$ network imaging protocols because the entire network appears to be recruited, based on evidence from current clamp and cell attached recordings (Trevelyan et al., 2006). Of course these recordings are susceptible to sampling bias, but this is also consistent with $c$-fos staining of post-epileptic tissue (Morgan et al., 1987), which samples the network more comprehensively. Furthermore, given the intensity of synaptic bombardment at this time, and the progressive failure of inhibition, it seems inconceivable that neurons do not fire during some stage of the event (even if some cells may 
1 experience periods of restraining inhibition (Trevelyan et al., 2006), or subsequently enter depolarising blockade (Cammarota et al., 2013; Ziburkus et al., 2006)). This gives us a very good positive control therefore, allowing us to focus only on cells that show increases in the $\mathrm{Ca}^{2+}$ signal at this time (Trevelyan and Yuste, 2009). In such preparations there are small numbers of cells which are very brightly labelled throughout, and do not show fluctuations, but these typically lie close to the surface of the brain slice, and under differential interference contrast (DIC) look very dysmorphic - these cells were presumed to be terminally injured during the slice preparation, and we disregarded these for analyses. Unfortunately, these do interfere with imaging of deeper located cells, so improvements in slice preparation technique can also improve the imaging data. This remains something of an art, but post-slicing treatment of the slices is likely to be paramount. Finally, it is worth noting that the GUI could also be used to match fixed immunohistochemical data to live images taken in vivo.

In this study, we focused on the activity patterns of PV cells during a seizure event. While it is known from optogenetic studies that stimulation of PV cells can mediate the late discharges following the ictal wave front (Ellender et al., 2014), other studies have shown that interneurons can enter a period of depolarising block after the onset of seizures (Cammarota et al., 2013; Ziburkus et al., 2006). Notably both these studies induce epileptiform activity using the $\mathrm{K}^{+}$ channel blocker, 4-aminopyridine (in addition to $0 \mathrm{Mg}^{2+}$ ), which specifically compromises the facility of fast-spiking interneurons to repolarise. It had remained unclear, therefore, whether this population of interneurons participate naturally during the late discharges, when 4aminopyridine was not present. Our new methodology allowed us to derive activity profiles for multiple PV interneurons, and show that many of these cells do indeed show sharp upward deflections of intracellular $\mathrm{Ca}^{2+}$ at the start of successive late discharges, indicative of intense bursting during these events. This highlights a subtle, but notable difference in how different pharmacological manipulations impact on network activity.

\section{Acknowledgments}

We would like to thank Rolando Berlinguer-Palmini and Alex Laude, in the Bio-Imaging Unit at Newcastle University, for their help with microscopy.

This work was supported by Epilepsy Research UK [P1504 to A.J.T, C.R and R.R.P] and the 
1 Doctoral Training Programme (IoN); N.C. was supported by Medical Research Council 2 (MR/J013250/1 to A.J.T. and C.R.)

\section{Figure legends}

4 Fig. 1. Flow chart illustrating the methodology.

5 Fig. 2. Electrophysiology and $\mathrm{Ca}^{2+}$ imaging can be used to follow patterns of network activity with cellular resolution. (A) Patch-clamp electrophysiology recording of a neocortical pyramidal cell being used to determine when the cell and the surrounding neocortical tissue were experiencing pre-ictal, ictal, and post-ictal activity. (B) Snapshots of the field of view at particular time points of the $\mathrm{Ca}^{2+}$ network imaging (see $\mathrm{C}$ ) (pre-ictal, $\mathrm{t}=6.3$ and $15.8 \mathrm{~ms}$; ictal, $\mathrm{t}=19$ and $25.3 \mathrm{~ms}$ ). The dashed lines indicate the patch pipette for the cell recording in (A). (C) Raster plot of the $\mathrm{Ca}^{2+}$ network imaging signalling for the same time window as the electrophysiology recording, at the same cortical location. Each row of pixels shows the fluorescence level for a single neuron $(n=113$; grayscale has arbitrary units: centresurround signal (Trevelyan et al., 2006)).

Fig. 3. Characterising the live imaging landmarks. (A) Projection of a live z-stack of the patched pyramidal cell that was filled with Alexa Fluor 594. (B) Projection of the live z- stack of cells in the field of view of the patched cell in (A) that were imaged using either a $\mathrm{Ca}^{2+}$ dye or a genetically encoded calcium sensor. Arrow indicates patched cell in (A). (C) Low and (D) high magnification images of the tissue orientation within the recording chamber; the images were taken at the end of the experiment before fixing the tissue.

Fig. 4. Matching of live and fixed tissue imaging. 1. Imaging: (A) Projection of a $\mathrm{z}$-stack of live images of the recorded cell (green) surrounded by glia cells labelled with SR101(red). (Ai) Projection of a z-stack of live images of cells filled with the $\mathrm{Ca}^{2+}$ dye OGB (green). (B) Projection of a z-stack of fixed images of same cell in (A), with the neuron labelled with Streptavidin (green) and the glia cells labelled with an antibody against GFAP (red). (Bi) Projection of a z-stack of fixed images of cell labelled with NeuroTrace. The image is for the same field of view (ROI) and has the same orientation of (A-B).

2. Landmarks: (Aii) Projection of the z-stack of live imaging of the Alexa Fluor filled cell and the ROI with example landmarks. Blue dots represent landmarks used to match it with the fixed image in (Bii). (Bii) Projection of the z-stack of fixed imaging of the biocytin-streptavidin cell 
1 and the ROI with example landmarks. Blue dots represent landmarks used to match with the

2 live image in (Aii).

3 3. Mapping: (Aiii) Projection of the z-stack of the live imaging used to match the projection of 4 the z-stack of the images from fixed tissue following linear distortion using matchgui (Biii).

5 Note that the coloured framed boxes are examples of the areas matched in the live (Aiii) and

6 fixed (Biii) tissue imaging. Note also that the distortion reduced the size of the fixed images,

7 illustrating nicely why the low-level alignment is important so as to get appropriate matching

8 while limiting the size of the files (i.e. the orientation of the slice on the microscope slide).

9 Note that the ROI in fixed imaging in (B,Bi,Bii,Biii) was taken at high magnification, and for

104 different partially overlapping fields of view, which were then tiled together. The mosaic of 11 multiple tiles was obtained by using the corresponding function of the Zeiss AxioVision 12 software.

13 (C) High magnification of the areas within the coloured framed boxes in (Aiii and Biii). The 14 areas contain matched cells from the overlay of the live (Aiii) and fixed (Biii) images, generated 15 in matchgui.

17 Figure 5. Comparison of different transformation algorithms (A) Initial input images for the matchgui software, with the matched landmarks shown as blue spots on both the source (Ai, fixed tissue, compressed image of the filled cell (red) and the NeuroTrace label at what was identified as the equivalent $\mathrm{z}$ plane as the functional data set) and target (Aii, standard deviation compression of a time series of $\mathrm{Ca}^{2+}$ network imaging during an epileptiform event) images. Note that the source images were created from an extended montage aimed at extending beyond the field of view of the live images (target).

(B) Three different transformations based on the landmarks. (C) Zoomed in views of equivalent regions of the 3 transformed imaged, and also of the live image. Note how in the central region, all three transformations match the live imaging, and also match with each other. At the periphery of the image, however, the best match to the live imaging comes from the LWM transformation.

Figure 6. The distribution of identified cells is weighted heavily to the centre of the field of view. (A) A standard-deviation compression of a time series of $\mathrm{Ca}^{2+}$ network imaging data. (B) Cells which showed fluctuations of signal above the background neuropil signal, indicative 
1 of activation during the epileptiform event (Trevelyan et al., 2006). (C) Cells are highlighted 2 in red, for which we acquired firing pattern data, and which could be precisely matched to the

3 fixed cells. Note that while only about $50 \%$ of the cells with functional signals were matched,

4 within the central area, where there were the most matching landmarks, close to $100 \%$ are

5 matched. (D-F) Equivalent series of images from a different slice, in which $\mathrm{Ca}^{2+}$ network

6 imaging was performed using the genetically engineered $\mathrm{Ca}^{2+}$ indicator, GCaMP6f.

Fig. 7. Genetically encoded calcium sensors can be used to match up live versus fixed tissue imaging, with cell class identification. (A) Single-plane-of-focus image, made by compressing a movie time series into a single image (standard deviation stack compression), from live images of a seizure event captured using GCaMP6f (see also Fig. 9). (B) Linear distortion produced in matchgui of a single plane of focus from a z-stack from fixed tissue imaging matched to the live imaging in (A). GCaMp6f (green), NeuroTrace (blue), and PV (red). (C) High magnifications of the coloured framed boxes in (A) and (B) showing the matching of post-hoc identified neurons to the live imaging. This allows the identification of which cells are PV positive in the live imaging. Note that being these single-plane-of-focus images, the PV labelling only partially define the whole soma of the positive cells. These images were not optimised for brightness or contrast.

Figure 8. Concurrent and separate visualisation of 5 different chromofluors contained within the fixed brain slice. (A) Biocytin labelling of the filled cell; (B) NeuroTrace labelling; immunoreactivity against somatostatin $(\mathrm{C})$, parvalbumin composite image, showing all 5 markers together.

Fig. 9. Identification of PV cell activity during a seizure event. (A) Projection of the $\mathrm{Ca}^{2+}$ network activity (standard deviation projection of a movie), with the PV cells outlined in red, as identified from the matching methodology in Fig. 7. Subsequent panels show active cells at given time points during the evolving epileptiform event. (B) Raster plot of the 161 identified cells from the $\mathrm{Ca}^{2+}$ network imaging, aligned according to their largest step change in $\mathrm{Ca}^{2+}$ 
1 recording of the pyramidal cell during the event. (D) Raster plots, showing the activity of the

$27 \mathrm{PV}$ interneurons, derived from thresholding the first derivative of the $\mathrm{Ca}^{2+}$ signal for these

3 cells. Note the very prominent early activity of several of these neurons, and also the evidence

4 of firing continuing throughout the event. (B,C,D) same temporal scale bar $(10 \mathrm{sec})$.

\section{$5 \quad$ References}

6 Abe T, Fujimori T. Reporter mouse lines for fluorescence imaging. Development, growth \& 7 differentiation, 2013; 55: 390-405.

8 Baird GS, Zacharias DA, Tsien RY. Circular permutation and receptor insertion within green

9 fluorescent proteins. Proceedings of the National Academy of Sciences of the United States of

10 America, 1999; 96: 11241-6.

11 Cammarota M, Losi G, Chiavegato A, Zonta M, Carmignoto G. Fast spiking interneuron 12 control of seizure propagation in a cortical slice model of focal epilepsy. J Physiol, 2013; 591: 13 807-22.

14 Cossart R, Aronov D, Yuste R. Attractor dynamics of network UP states in the neocortex. 15 Nature, 2003; 423: 283-8.

16 Ellender TJ, Raimondo JV, Irkle A, Lamsa KP, Akerman CJ. Excitatory effects of 17 parvalbumin-expressing interneurons maintain hippocampal epileptiform activity via 18 synchronous afterdischarges. J Neurosci, 2014; 34: 15208-22.

19 Fujiwara-Tsukamoto Y, Isomura Y, Nambu A, Takada M. Excitatory gaba input directly drives 20 seizure-like rhythmic synchronization in mature hippocampal CA1 pyramidal cells. 21 Neuroscience, 2003; 119: 265-75.

22 Grynkiewicz G, Poenie M, Tsien RY. A new generation of Ca2+ indicators with greatly improved fluorescence properties. The Journal of biological chemistry, 1985; 260: 3440-50. Gutierrez R, Armand V, Schuchmann S, Heinemann U. Epileptiform activity induced by low $\mathrm{Mg} 2+$ in cultured rat hippocampal slices. Brain research, 1999; 815: 294-303.

Lewandoski M. Conditional control of gene expression in the mouse. Nature reviews. Genetics, 2001; 2: 743-55.

Lin MZ, Schnitzer MJ. Genetically encoded indicators of neuronal activity. Nature neuroscience, 2016; 19: 1142-53.

Losi G, Marcon I, Mariotti L, Sessolo M, Chiavegato A, Carmignoto G. A brain slice experimental model to study the generation and the propagation of focally-induced epileptiform activity. Journal of neuroscience methods, 2016; 260: 125-31.

Losi G, Mariotti L, Sessolo M, Carmignoto G. New Tools to Study Astrocyte Ca2+ Signal Dynamics in Brain Networks In Vivo. Frontiers in cellular neuroscience, 2017; 11: 134.

MacLean JN, Watson BO, Aaron GB, Yuste R. Internal dynamics determine the cortical response to thalamic stimulation. Neuron, 2005; 48: 811-23.

Miyawaki A, Llopis J, Heim R, McCaffery JM, Adams JA, Ikura M, Tsien RY. Fluorescent indicators for $\mathrm{Ca} 2+$ based on green fluorescent proteins and calmodulin. Nature, 1997; 388: $882-7$.

40 Mody I, Lambert JD, Heinemann U. Low extracellular magnesium induces epileptiform 41 activity and spreading depression in rat hippocampal slices. J Neurophysiol, 1987; 57: 869-88. 42 Morgan JI, Cohen DR, Hempstead JL, Curran T. Mapping patterns of c-fos expression in the central nervous system after seizure. Science, 1987; 237: 192-7.

Muzumdar MD, Tasic B, Miyamichi K, Li L, Luo L. A global double-fluorescent Cre reporter mouse. Genesis, 2007; 45: 593-605. 
1 Nakai J, Ohkura M, Imoto K. A high signal-to-noise $\mathrm{Ca}(2+)$ probe composed of a single green

2 fluorescent protein. Nature biotechnology, 2001; 19: 137-41.

3 Nimmerjahn A, Kirchhoff F, Kerr JN, Helmchen F. Sulforhodamine 101 as a specific marker 4 of astroglia in the neocortex in vivo. Nat Methods, 2004; 1: 31-7.

5 Orban PC, Chui D, Marth JD. Tissue- and site-specific DNA recombination in transgenic mice.

6 Proceedings of the National Academy of Sciences of the United States of America, 1992; 89:

$7 \quad 6861-5$.

8 Parrish RR, Grady J, Codadu NK, Racca C, Trevelyan AJ. Graphical user interface for

9 simultaneous profiling of activity patterns in multiple neuronal subclasses. Data in Brief, 2018;

10 In review.

11 Powell SK, Rivera-Soto R, Gray SJ. Viral expression cassette elements to enhance transgene 12 target specificity and expression in gene therapy. Discovery medicine, 2015; 19: 49-57.

13 Rodriguez EA, Campbell RE, Lin JY, Lin MZ, Miyawaki A, Palmer AE, Shu X, Zhang J, Tsien 14 RY. The Growing and Glowing Toolbox of Fluorescent and Photoactive Proteins. Trends in 15 biochemical sciences, 2017; 42: 111-29.

16 Sauer B, Henderson N. Site-specific DNA recombination in mammalian cells by the Cre 17 recombinase of bacteriophage P1. Proceedings of the National Academy of Sciences of the 18 United States of America, 1988; 85: 5166-70.

19 Smetters D, Majewska A, Yuste R. Detecting action potentials in neuronal populations with 20 calcium imaging. Methods, 1999; 18: 215-21.

21 Trevelyan AJ, Baldeweg T, van Drongelen W, Yuste R, Whittington M. The source of 22 afterdischarge activity in neocortical tonic-clonic epilepsy. J Neurosci, 2007a; 27: 13513-9.

Trevelyan AJ, Schevon CA. How inhibition influences seizure propagation. Neuropharmacology, 2013; 69: 45-54.

Trevelyan AJ, Sussillo D, Watson BO, Yuste R. Modular propagation of epileptiform activity: evidence for an inhibitory veto in neocortex. J Neurosci, 2006; 26: 12447-55.

Trevelyan AJ, Sussillo D, Yuste R. Feedforward inhibition contributes to the control of epileptiform propagation speed. J Neurosci, 2007b; 27: 3383-7.

Trevelyan AJ, Yuste RM. Imaging seizure propagation in vitro. In Baraban SC, editor. Animal Models of Epilepsy: Methods and Innovations. Humana Press, 2009: 1051-6.

31 Vigouroux RJ, Belle M, Chedotal A. Neuroscience in the third dimension: shedding new light 32 on the brain with tissue clearing. Molecular brain, 2017; 10: 33.

33 Yang W, Yuste R. In vivo imaging of neural activity. Nature methods, 2017; 14: 349-59.

34 Yuste R, Peinado A, Katz LC. Neuronal domains in developing neocortex. Science, 1992; 257: $35 \quad 665-9$.

36 Ziburkus J, Cressman JR, Barreto E, Schiff SJ. Interneuron and pyramidal cell interplay during 37 in vitro seizure-like events. J Neurophysiol, 2006; 95: 3948-54. 


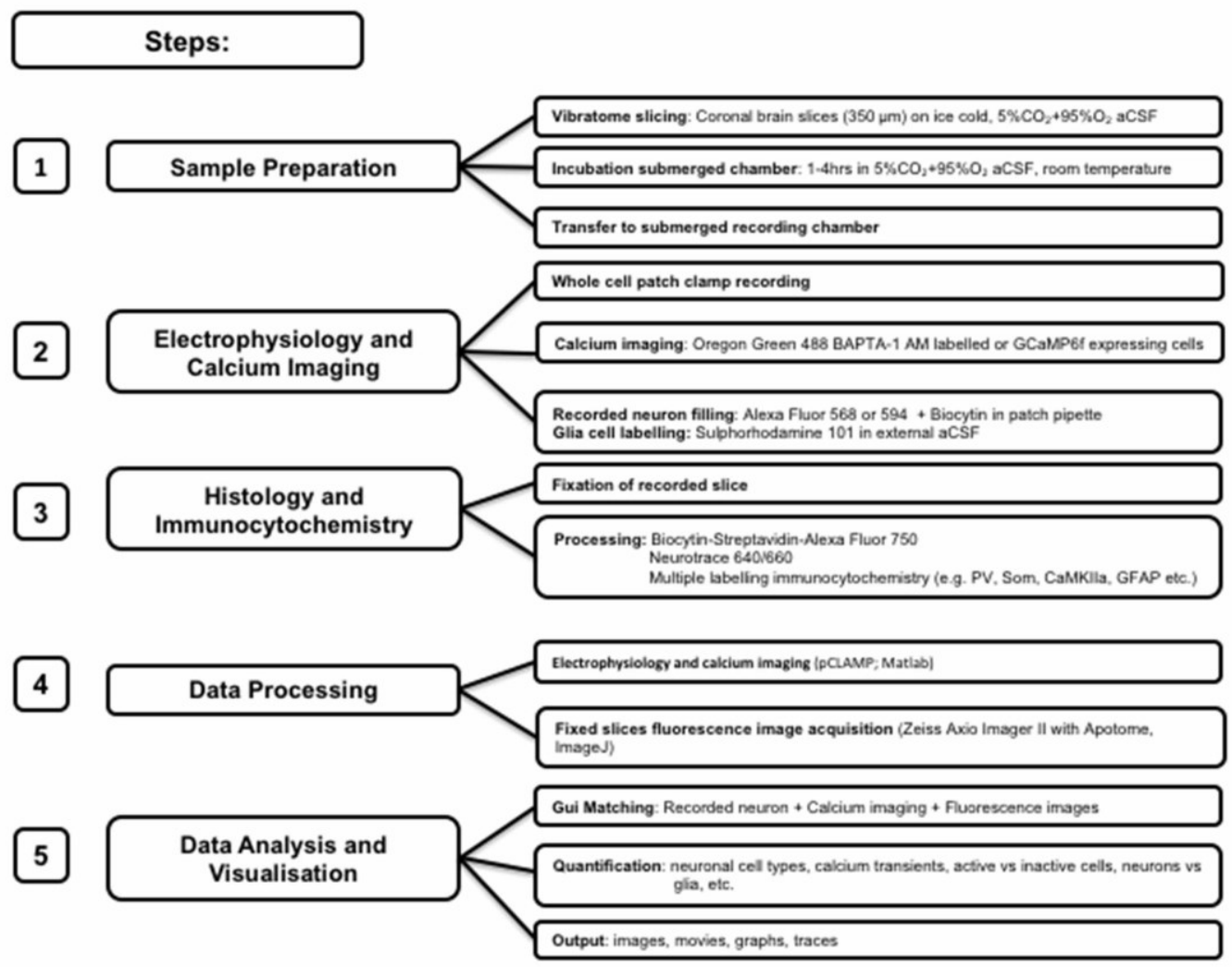



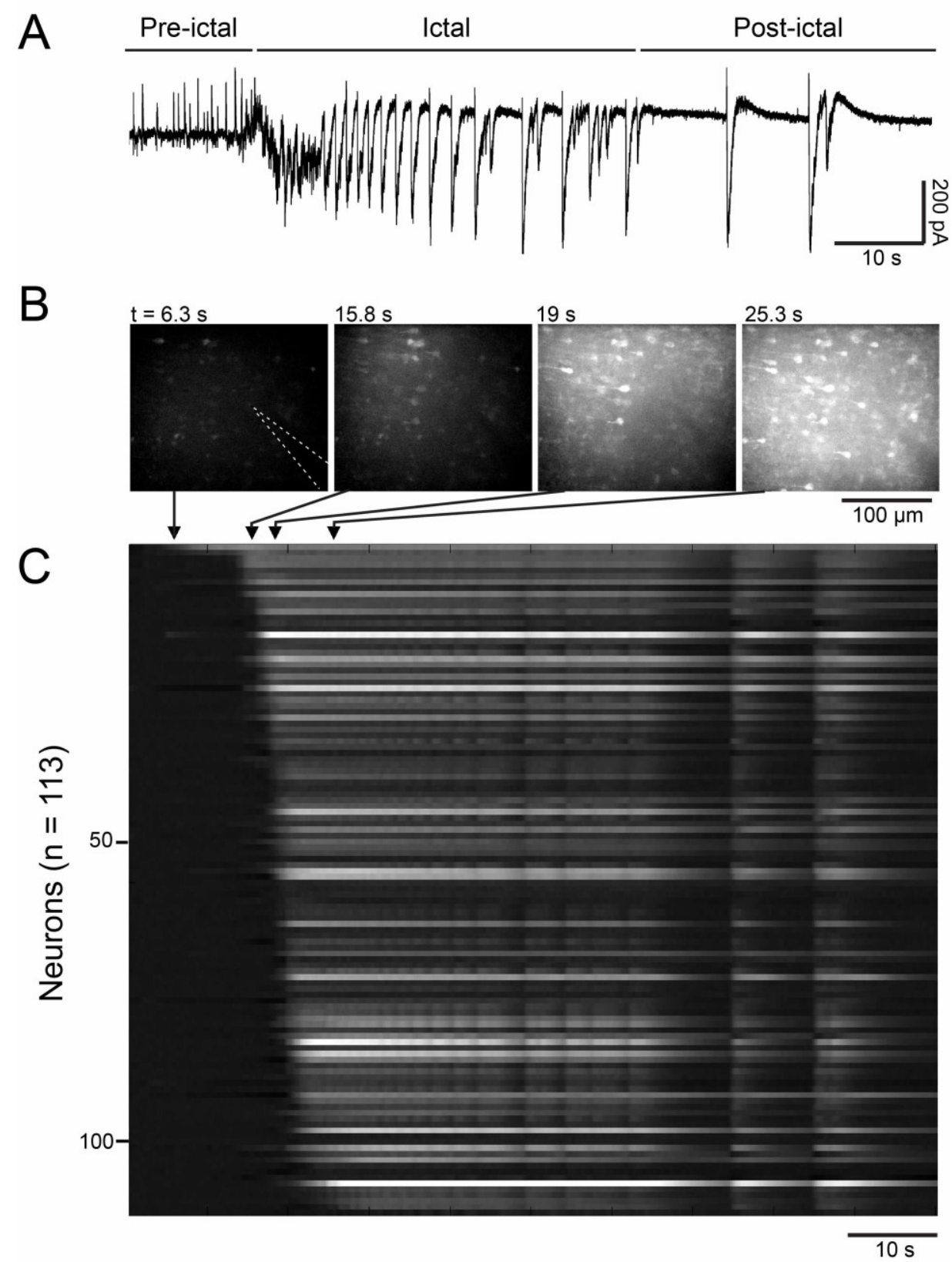

Figure 2 - Parrish et al. 


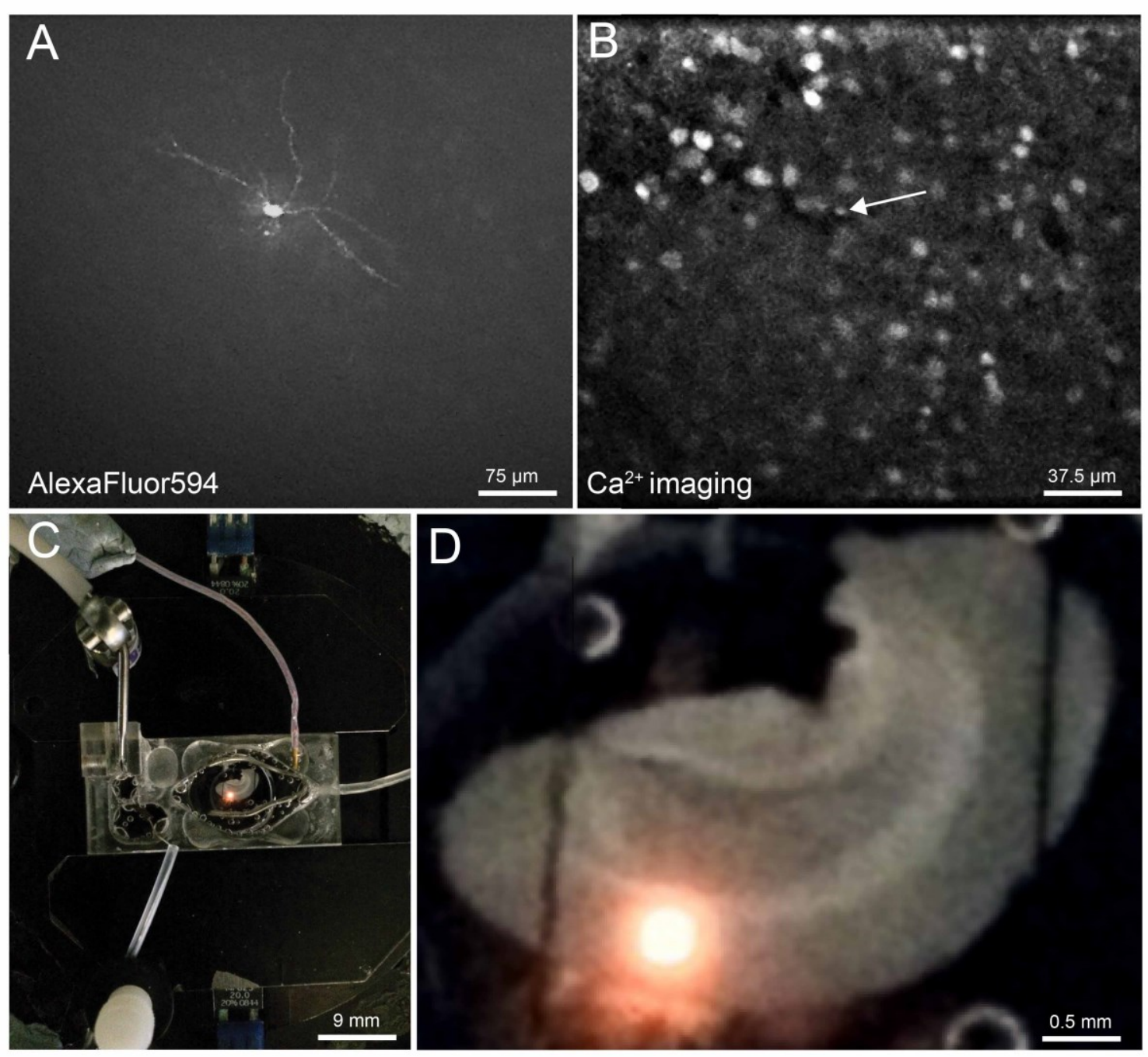

Figure 3 - Parrish et al. 
1. Imaging

Live Tissue
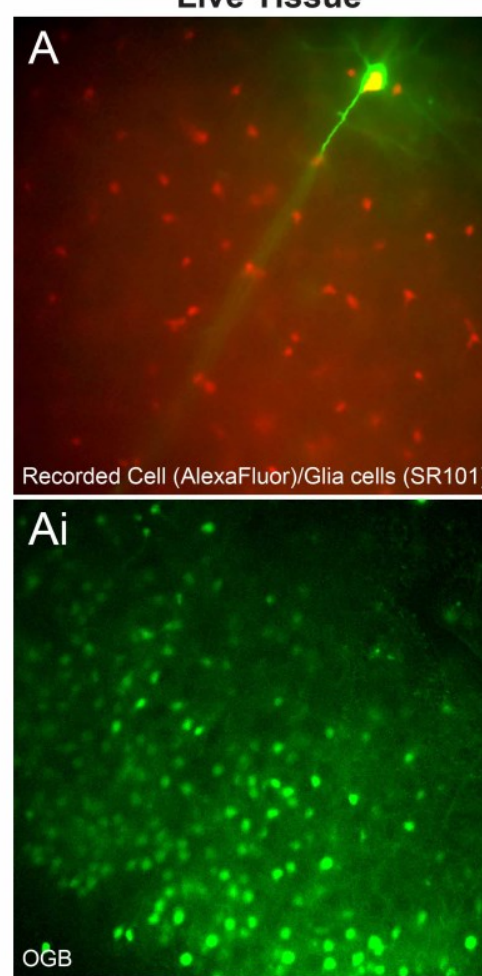

2. Landmarks

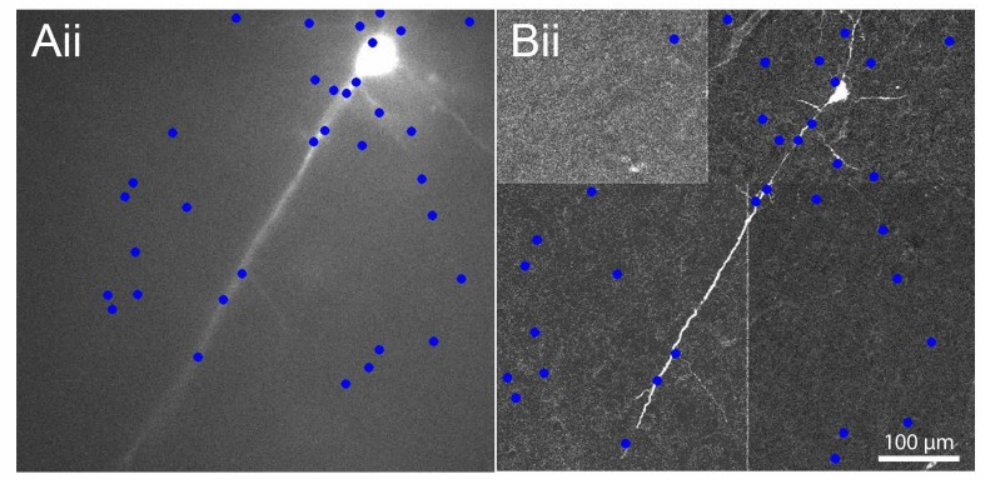

\section{Mapping}

Aiii Live Imaging (OGB)

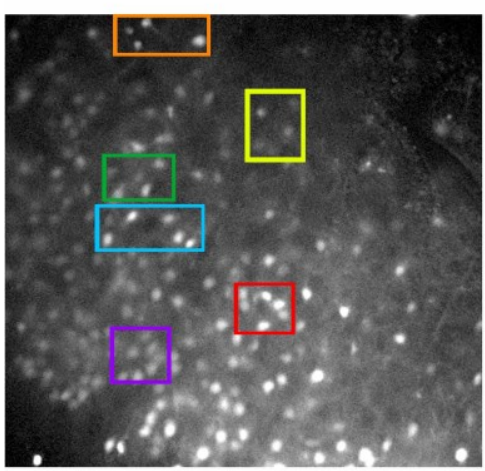

C

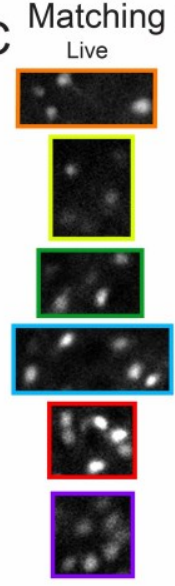

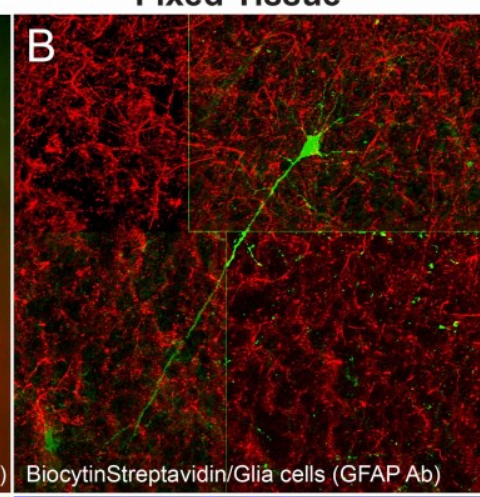

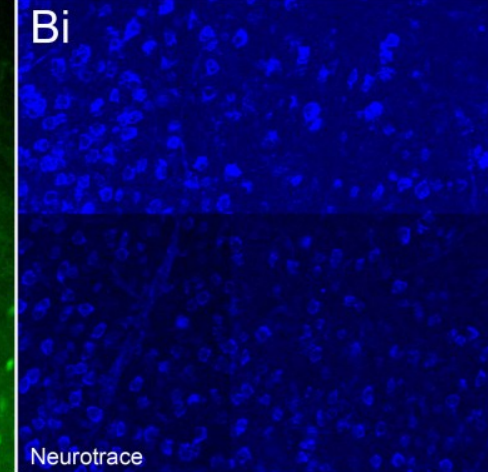

Neurotrace

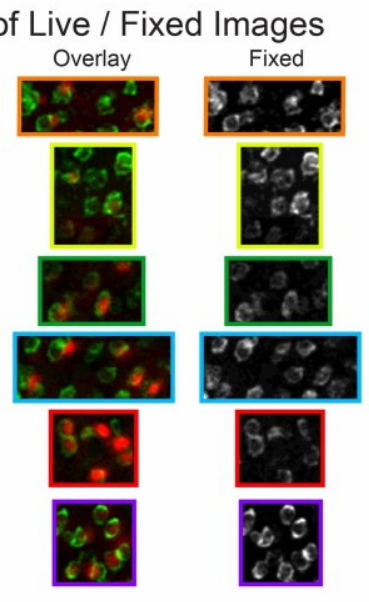

Biil Linear Distortion

Biii Fixed Tissue (Neurotrace)

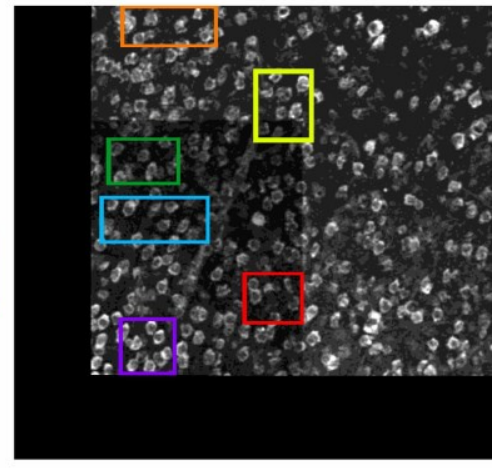

Figure 4 - Parrish et al 
A. Original images

Ai. Fixed tissue ("source")

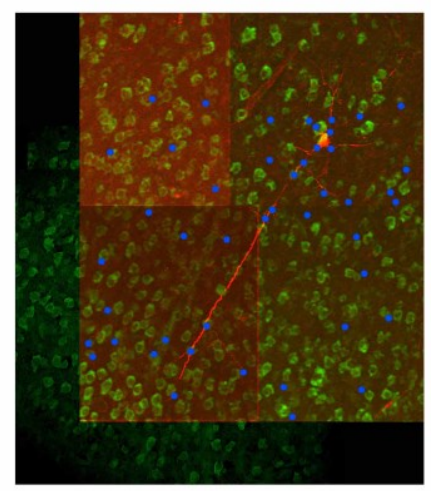

Aii. Live image ("target")

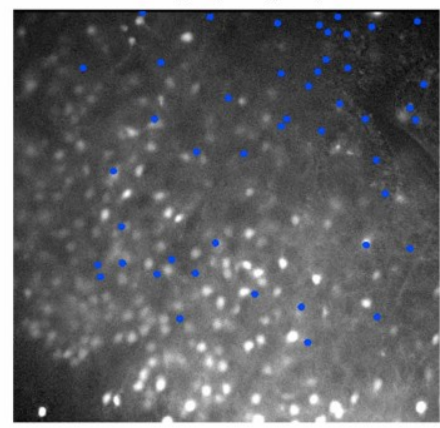

B. Transformed images

Bi.Linear conformal (LC)

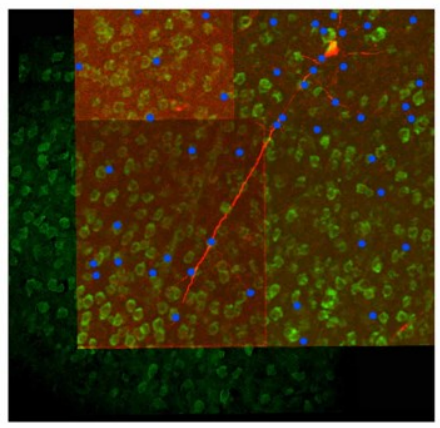

Biii. Local weighted mean (LWM)

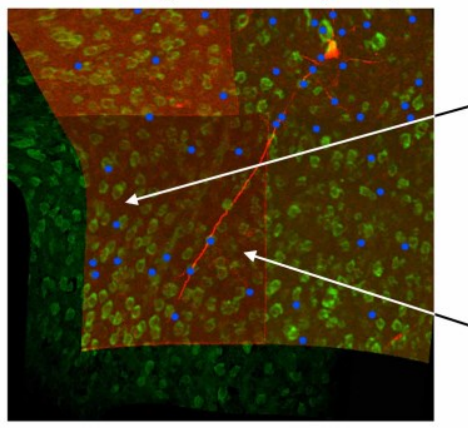

Bii. Piecewise $(P)$

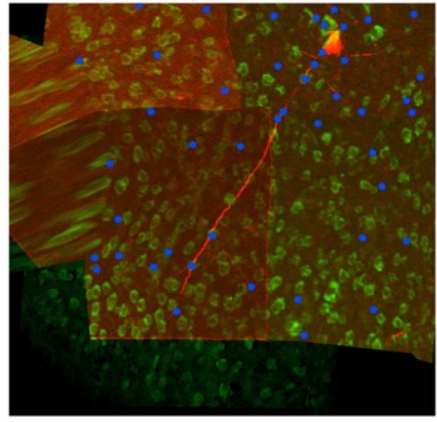

C. Example territories

Peripheral - displaced matches

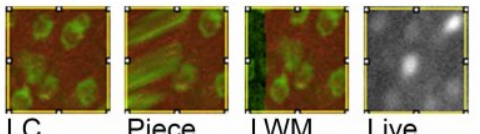

Central - good match

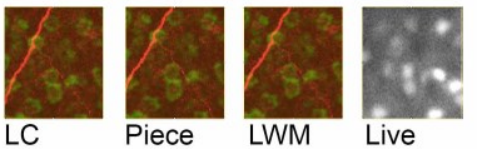

Figure 5 - Parrish et al 
A. Imaging time series compression (OGB1)

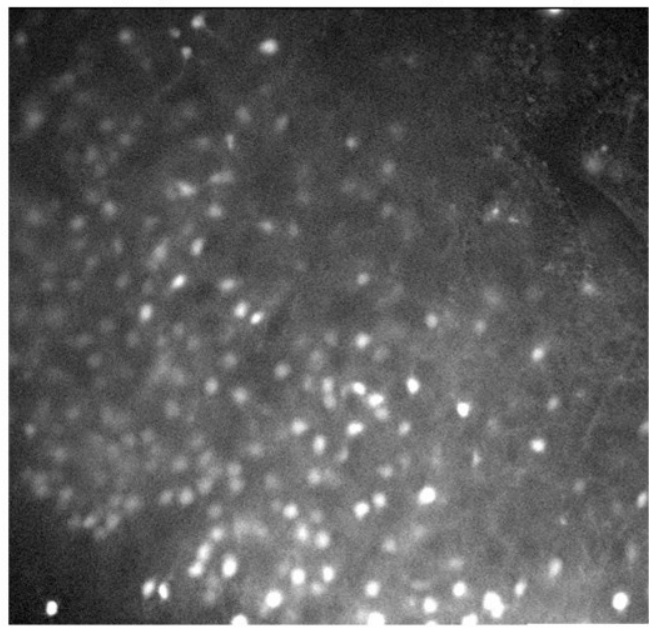

B. 247 cells with functional signal

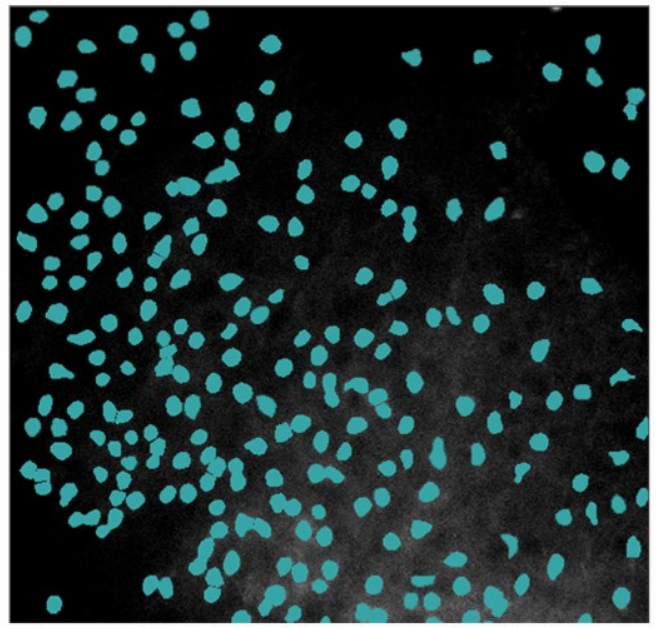

C. 120 cells identified in fixed images

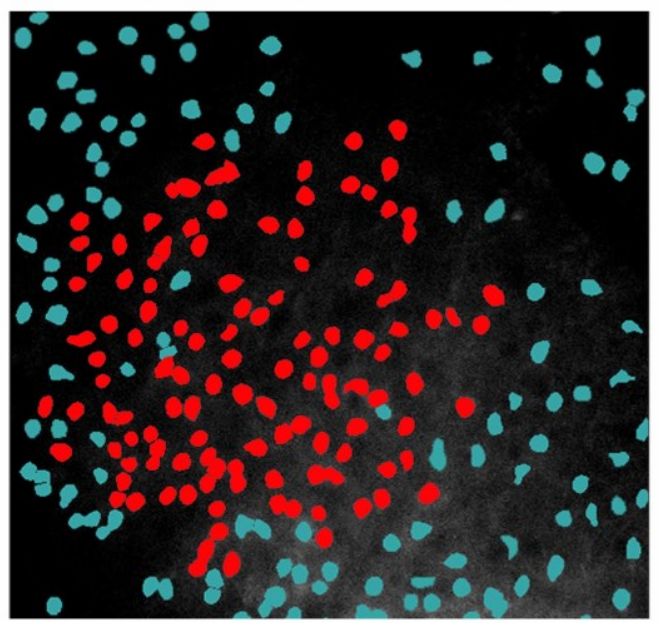

D. Time series compression (GCaMP6f)

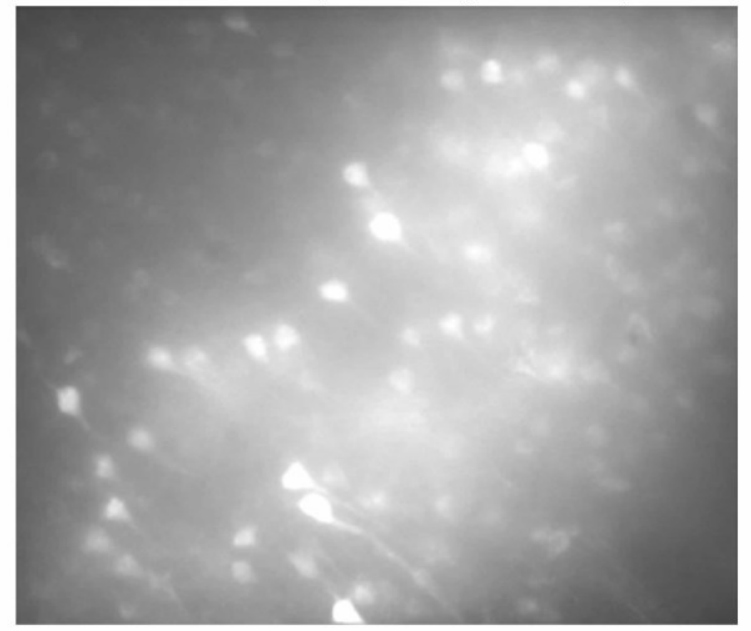

E. 166 cells with functional signal

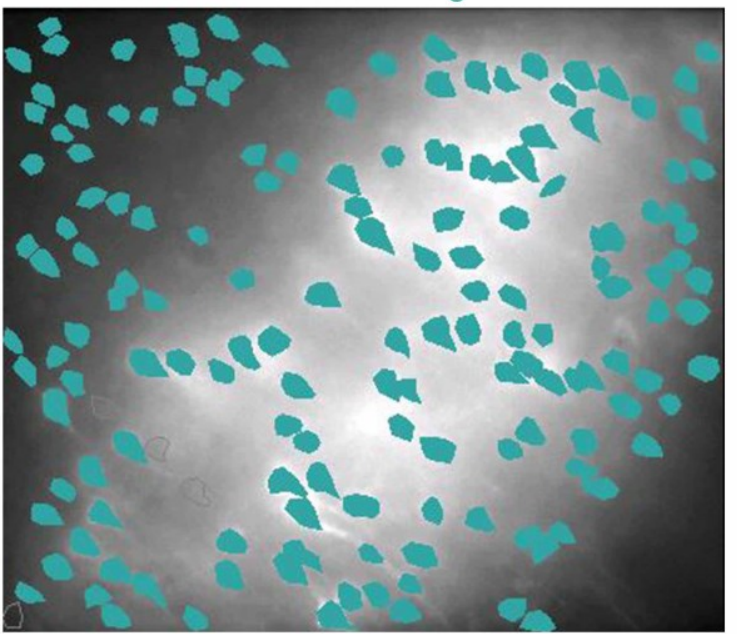

F. 103 cells identified

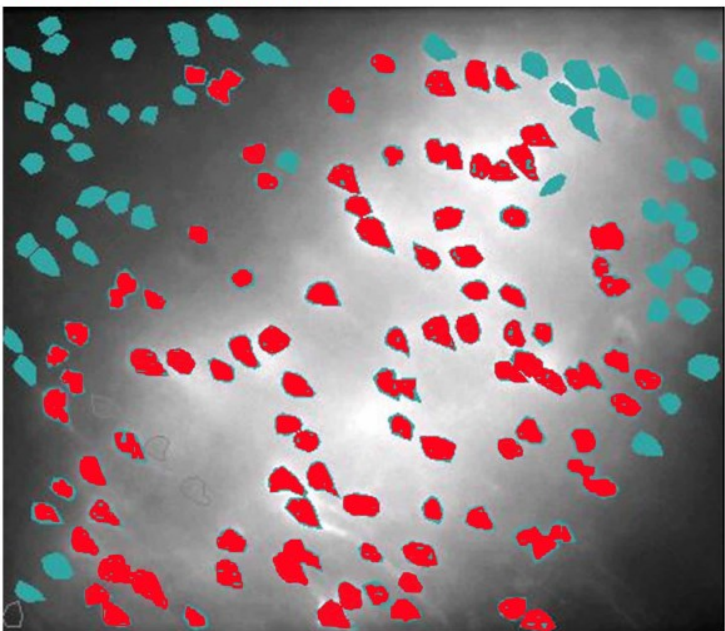

Figure 6 - Parrish et al. 


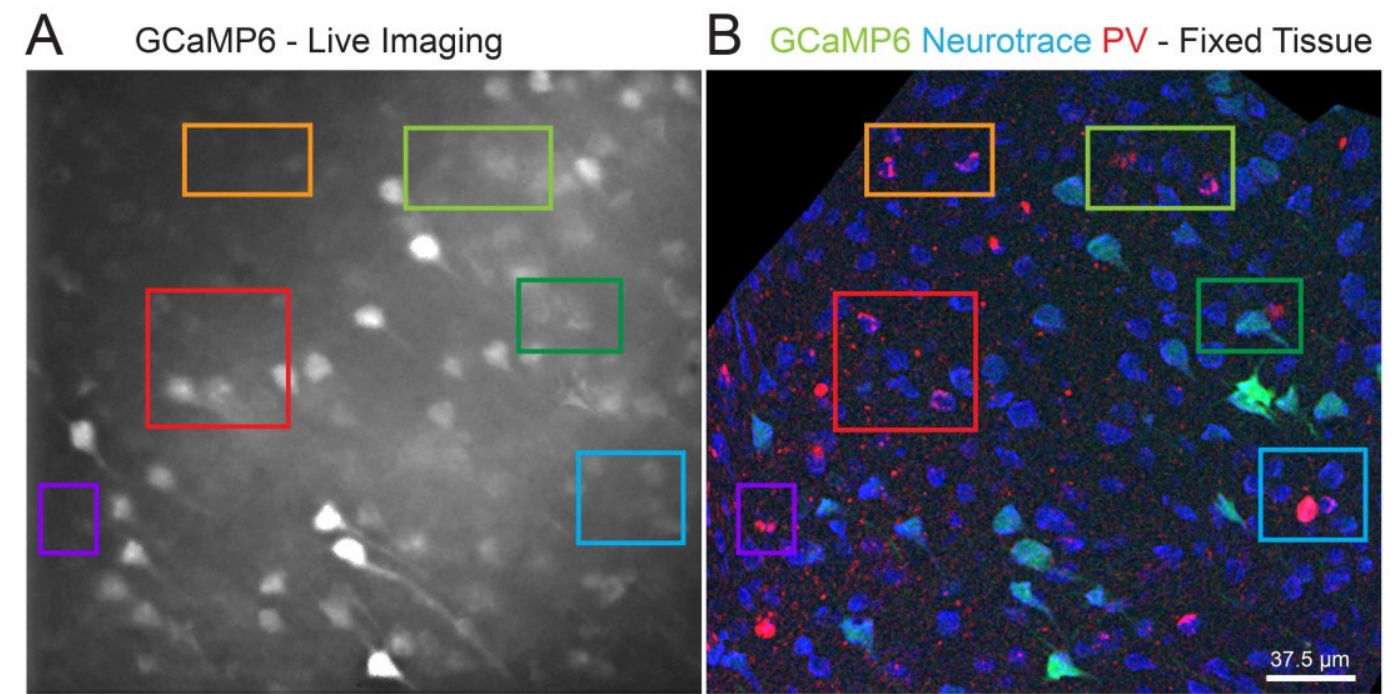

\section{GCaMP6 - Live GCaMP6 - Fixed Neurotrace - Fixed PV - Fixed}

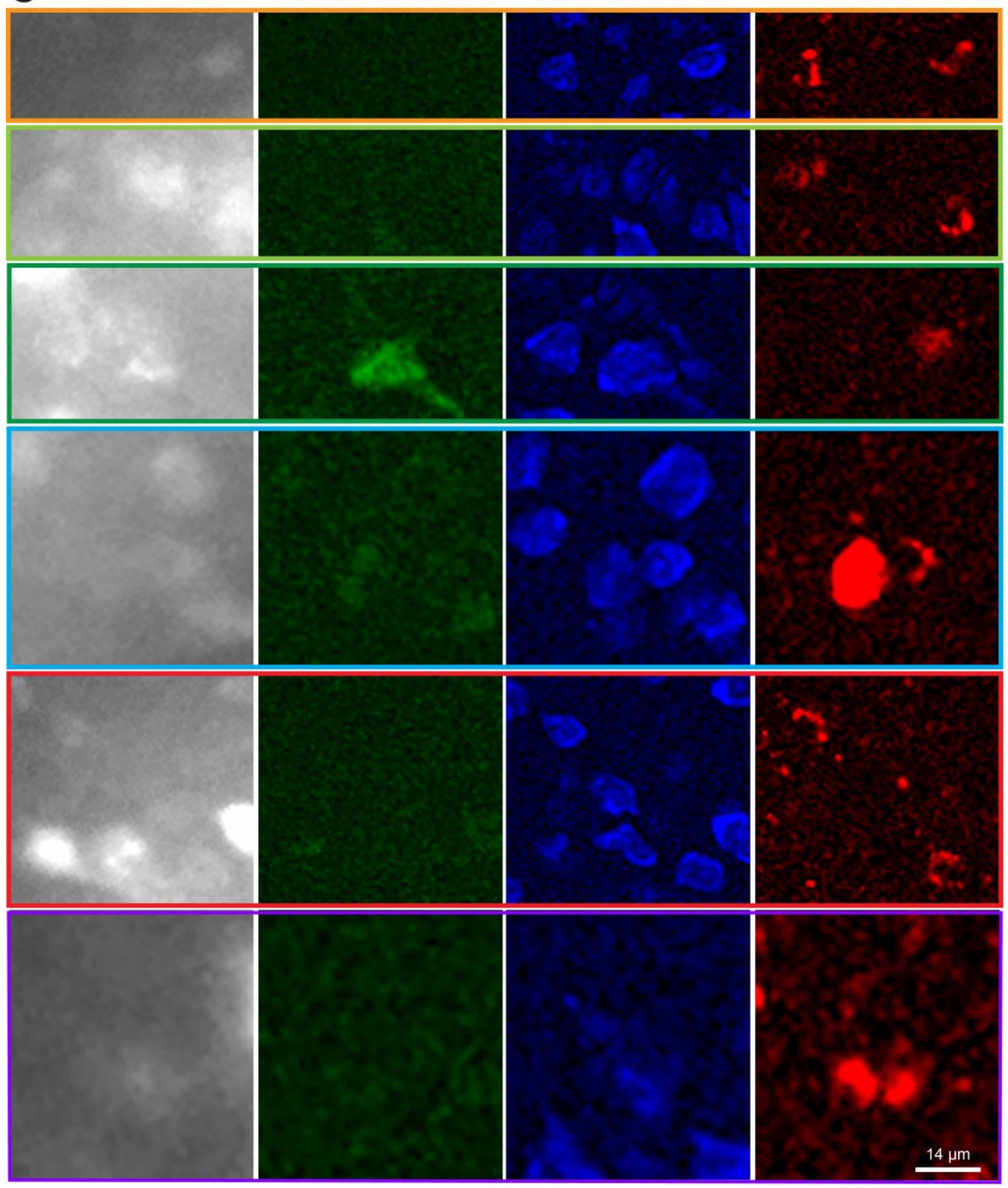

Figure 7 - Parrish et al. 


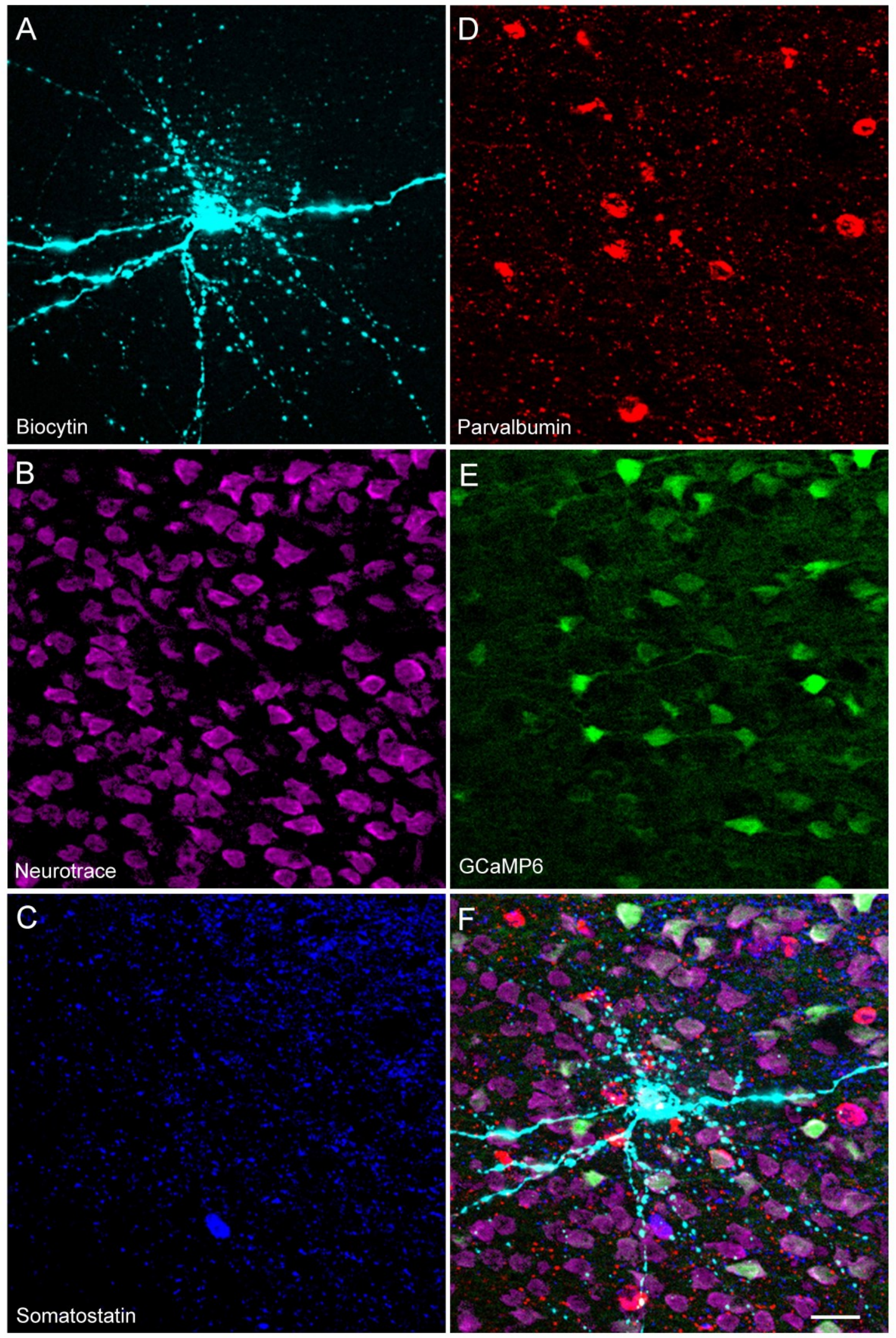




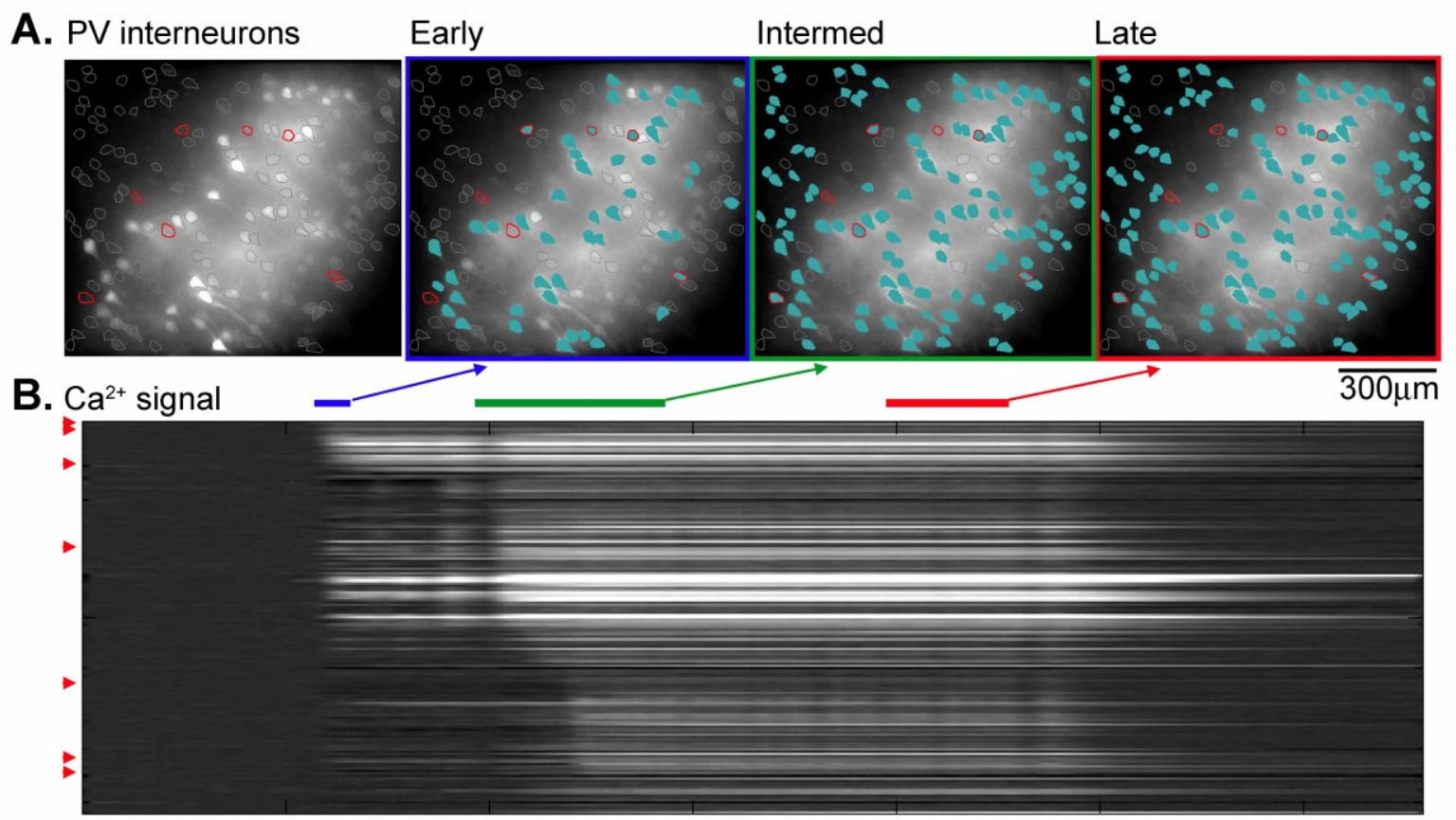

C. Pyramidal cell, voltage clamp, $-30 \mathrm{mV}$

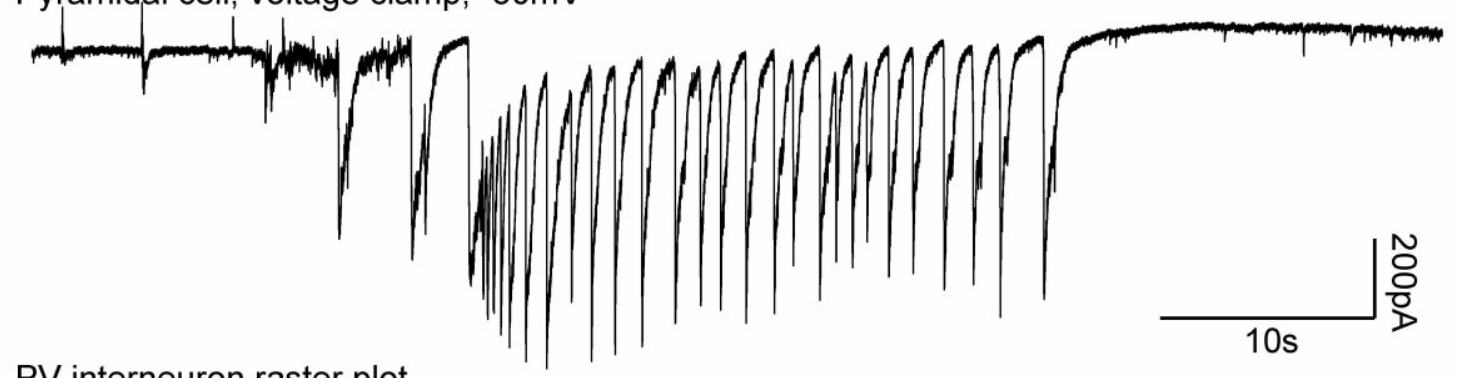

D. PV interneuron raster plot

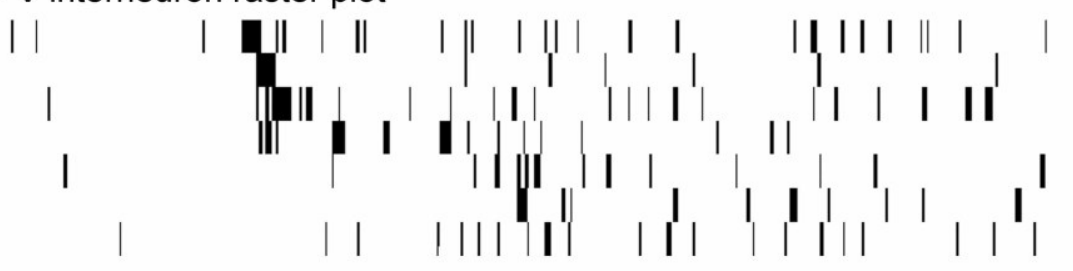

Figure 9 - Parrish et al. 